THE GLOBAL BOURGEOISIE 



\section{The Global Bourgeoisie}

THE RISE OF THE MIDDLE CLASSES

IN THE AGE OF EMPIRE

\section{EDITED BY CHRISTOF DEJUNG, \\ DAVID MOTADEL \& \\ JÜRGEN OSTERHAM MEL}


Copyright (C) 2019 by Princeton University Press

Published by Princeton University Press

41 William Street, Princeton, New Jersey o8540

6 Oxford Street, Woodstock, Oxfordshire OX20 1TR

press.princeton.edu

All Rights Reserved

LCCN 2019936039

ISBN 9780691195834

ISBN 9780691177342 (pbk.)

British Library Cataloging-in-Publication Data is available

Editorial: Eric Crahan, Thalia Leaf, and Pamela Weidman

Production Editorial: Debbie Tegarden

Cover design: Chris Ferrante

Cover credit: Pier of the Hotel de los Pocitos, Montevideo, Uruguay

Production: Erin Suydam

Publicity: Alyssa Sanford

Copyeditor: Gail K. Schmitt

This book has been composed in Arno Pro

Printed on acid-free paper. $\infty$

Printed in the United States of America

$\begin{array}{llllllllll}10 & 9 & 8 & 7 & 6 & 5 & 4 & 3 & 2 & 1\end{array}$ 